\title{
A Cardiac Graft from a Donor with Granulomatosis with Polyangiitis-A Case Report
}

\author{
Katharina Huenges ${ }^{1}$ Bernd Panholzer ${ }^{1}$ Jochen Cremer $^{1}$ Assad Haneya ${ }^{1}$ \\ ${ }^{1}$ Department of Cardiovascular Surgery, University of Schleswig- \\ Holstein, Campus Kiel, Kiel, Germany \\ Thorac Cardiovasc Surg Rep 2017;6:e3-e4.

\begin{abstract}
Address for correspondence Katharina Huenges, MD, Department of Cardiovascular Surgery, University of Schleswig-Holstein Campus Kiel, Arnold-Heller-Straße 3, Hs 18, Kiel 24105, Germany
\end{abstract} \\ (e-mail: Katharina.Huenges@uksh.de).
}

\author{
Abstract \\ Keywords \\ - systemic \\ - transplantation \\ - heart \\ - heart failure
}

Organ shortage unavoidably leads to shifting strategies in modern transplantation medicine. Experiences with specific comorbidities in terms of organ transplantation therefore have to be made. We report a case of a 51-year-old male patient with successful orthotopic heart transplantation from a donor with granulomatosis with polyangiitis. After a good recovery, the patient was discharged to rehabilitation 2 months after transplantation.

\section{Introduction}

Granulomatosis with polyangiitis (GPA) is a form of a systemic necrotizing vasculitis, affecting small and medium vessels in various organs. Mostly, lung, paranasal sinusoids, and renal (glomerulonephritis) affections are of clinical relevance for the patients, but it may also affect the heart. ${ }^{1-3}$ Circulating antineutrophil cytoplasmic antibodies in blood may lead to the diagnosis, definitely diagnosed via histological stains.

Transplantation medicine, particularly in Germany, is very much influenced by organ shortage. Certain comorbidities in organ donors are not yet well known in the context of potential organ transplantation.

This report presents a case of a patient with successful heart transplantation from a donor with GPA.

\section{Case Description}

We report a case of a 51-year-old male patient listed for heart transplantation with end-stage terminal heart failure due to dilative cardiomyopathy.

GPA was first diagnosed in a 20-year-old donor. Due to extended lung bleeding, the donor required extracorporeal membrane oxygenation (ECMO) support. Under ECMO support, a fatal subarachnoid bleeding occurred. After 5 days, the ECMO was successfully weaned. Echocardiography and coronary angiography revealed a good inconspicuous heart function under very low inotropic support. The patient was accepted as an organ donor according to the German Organ Transplantation Foundation and valid Eurotransplant guidelines.

After consulting our immunology department, no relative contraindication against transplanting a heart of a patient with GPA was found, since immunosuppression after transplantation was also considered to be effective in treating granulomatosis.

After the organ was accepted, orthotopic heart transplantation was performed. The cold ischemic time was 193 minutes.

An immediate stable sinus rhythm was achieved. The intraoperative transesophageal echocardiography showed an excellent biventricular function. The patient was transferred under low inotropic support and nitric oxygen (NO) insufflation toward our intensive care unit. The NO could be weaned within the first 5 hours; the catecholamine therapy could be substantially reduced, keeping epinephrine on a low-dose basis for 3 days. With sufficient pulmonary function, the patient was extubated 6 hours after the operation. No relevant rhythm disturbances occurred. Mobilization started immediately after removing the pulmonary catheter.

The immunosuppressive regimen in our institute is based on a triple-drug regimen including corticosteroids, mycophenolate mofetil, and calcineurin inhibitor (ciclosporin). An induction therapy with antithymocyte globulin was applied to the patient. Based on a cytomegalic virus status mismatch, an immune globulin therapy was conducted. received

August 19, 2016 accepted after revision October 24, 2016
DOI http://dx.doi.org/

10.1055/s-0036-1597597. ISSN 2194-7635. (c) 2017 Georg Thieme Verlag KG
Stuttgart · New York

License terms

(®) $\Theta \circledast$ 
Repeated echocardiography revealed normal left ventricular function and good right ventricular function without relevant valve insufficiency. The direct postoperative course was uneventful and free from major complications. With stable levels of immunosuppression therapy, stable cardiopulmonary function, and no of signs of infection, the patient was discharged to a rehabilitation clinic.

Endomyocardial biopsy was performed 25 days after the transplantation, with histopathological inconspicuous findings with no indication of rejection (International Society of Heart and Lung Transplantation, revised Allograft Rejection System, Grade 0, ISHLTOR).

\section{Discussion}

The number of organ donors continues to fall due to various reasons. This holds true particularly for Germany and stands in contrast to the increasing number of patients with end-stage terminal organ failure waiting for transplantation. This mismatch and organ shortage has led to postponing strategies in transplantation medicine. Despite improvements in the treatment of end-stage heart failure, heart transplantation is still considered as gold standard. Previous transplantation standards, ideals, and requirements are currently not entirely feasible. Acceptance of marginal organs (i.e., older donors, donors with comorbidities that were seen as mild contraindications for organ acceptance) remains low and very often those used to be considered as unsuitable for transplantation. However, under certain circumstances, they are becoming the only option for critically ill patients on the waiting list.

GPA, formerly known as Wegener disease, is rarely diagnosed. The few existing case reports focus on donors with GPA were found. Most research deals with GPA recipients requiring renal transplantation and possible reactivation after organ transplantation. Little is known about cardiac affection, transmission, or reactivation after solid organ transplantation.

In terms of cardiac involvement in GPA, the main concern is pericarditis. A few case reports describe pericardial masses, ${ }^{4}$ intracardial masses, ${ }^{1}$ and those rare intracardial masses may be associated with complete heart block. ${ }^{3}$ GPA might also mimic as left atrial myxoma. ${ }^{5}$ By the time of first granulomatosis diagnosis, the heart is affected in around
6 to $44 \%$ patients and, due to subclinical manifestations, might be often underestimated. ${ }^{1,2}$

In contrast to GPA, cardiac involvement in eosinophilic GPA (Churg-Strauss syndrome), a small vessel vasculitis, can occur more frequently ${ }^{6}$ and with more clinical relevance. ${ }^{7,8}$

Therapy regimens in patients with GPA are based on an immunosuppressive strategy, including steroids and cyclophosphamide combined with plasmapheresis ${ }^{3}$ or steroids with biologicals such as rituximab. ${ }^{4}$

In our case with dilatative cardiomyopathy, the orthotopic heart transplantation was successful and had an ordinary perioperative management. In conclusion, our experience suggests that the usage of heart organ from donors with GPA is possible and may lead to an excellent transplantation outcome without signs of granulomatosis disease in the recipient. However, the presented case is a single case experience, and therefore, further long-term studies are necessary to thoroughly understand both risks and benefits.

\section{References}

1 Mortazavi M, Nasri H. Granulomatosis with polyangiitis (Wegener's) presenting as the right ventricular masses: a case report and review of the literature. J Nephropathol 2012;1(1):49-56

2 Oliveira GH, Seward JB, Tsang TS, Specks U. Echocardiographic findings in patients with Wegener granulomatosis. Mayo Clin Proc 2005;80(11):1435-1440

3 Taskesen T, Goldberg SL, Mannelli L, et al. Granulomatosis with polyangiitis presenting with an intracardiac mass and complete heart block: enhanced images by 3-dimensional echocardiography. Circulation 2015;132(10):961-964

4 Sikorska D, Tykarski A, Radziemski A, Mojs E, Samborski W. Atypical location of granulomatosis with polyangiitis (Wegener's) with heart involvement-effectiveness of treatment with rituximab. Kardiol Pol 2015;73(12):1338

5 Herbst A, Padilla MT, Prasad AR, Morales MC, Copeland JG. Cardiac Wegener's granulomatosis masquerading as left atrial myxoma. Ann Thorac Surg 2003;75(4):1321-1323

6 Dennert RM, van Paassen P, Schalla S, et al. Cardiac involvement in Churg-Strauss syndrome. Arthritis Rheum 2010;62(2):627-634

7 Churg J, Strauss L. Allergic granulomatosis, allergic angiitis, and periarteritis nodosa. Am J Pathol 1951;27(2):277-301

8 Ramakrishna G, Connolly HM, Tazelaar HD, Mullany CJ, Midthun DE. Churg-Strauss syndrome complicated by eosinophilic endomyocarditis. Mayo Clin Proc 2000;75(6):631-635 